\title{
Stellung der Sentinel-Lymph-Node-Biopsie bei malignem Melanom
}

\section{The Role of Sentinel Lymph Node Biopsy of Malignant Melanoma}

Autor

Institut

\section{Y. Kröning}

Klinik für Dermatologie, Venerologie und Allergologie/Immunologisches Zentrum des Städtischen Klinikums Dessau

\section{Bibliografie}

DOI $10.1055 / s-2008-1077533$

Online-Publikation:

15. 10.2008

Akt Dermatol 2008; 34:

472-474 @ Georg Thieme

Verlag KG Stuttgart · New York ISSN 0340-2541

\section{Korrespondenzadresse}

\section{Dr. med. Yvonne Kröning}

Klinik für Dermatologie,

Venerologie und Allergologie/

Immunologisches Zentrum des

Städtischen Klinikums Dessau

Auenweg 38

06847 Dessau-Roßlau

Yvonne.kroening@

klinikum-dessau.de

\section{Zusammenfassung \\ $\nabla$}

Die Sentinel-Lymph-Node-Biopsie ist eine zuverlässige Stagingmethode und sollte bei Patienten mit malignem Melanom ab $1 \mathrm{~mm}$ Tumordicke durchgeführt werden. Die rezidivfreie Überlebenszeit von Melanompatienten korreliert eindeutig mit dem Status des Sentinel-Lymph-Node

\section{Einleitung}

Die Sentinel-Lymph-Node-Biopsie zählt heute als etablierte Methode des nodalen Stagings beim malignen Melanom ab einer Tumordicke von $1 \mathrm{~mm}$. Diese Methode wurde von Morton et al. 1992, damals noch unter Anwendung von 2,5\% Patentblau V zur intraoperativen Darstellung des Sentinel-Lymph-Node (SLN), eingeführt. Die SLN-Biopsie wurde entwickelt, um den ersten drainierenden Lymphknoten (LK) der regionalen Lymphabstromstation darzustellen. Dieser LK ist die wahrscheinlichste Lokalisation einer beginnenden Metastasierung [1].

Durch diese minimalinvasive operative Maßnahme ist es nun möglich, auf eine elektive Lymphknotendissektion zu verzichten. Die SLN-Biopsie ist eine grundsätzliche Methode des nodalen Stagings bei malignem Melanom. Auch bei nicht melanozytären High-risk-Malignomen (Merkelzellkarzinom, Plattenepithelkarzinom) ist die SLN-Biopsie indiziert [2-4].

\footnotetext{
* Nach einem Vortrag gehalten anlässlich des Symposions „Moderne Aspekte der Dermatoonkologie“ organisiert vom Tumorzentrum und der Klinik für Dermatologie, Venerologie und Allergologie - Immunologisches Zentrum, Städtisches Klinikum Dessau, März 2007.
}

(SLN). In zahlreichen klinischen Studien wurde gezeigt, dass der SLN eine wichtige diagnostische und prognostische Bedeutung für Patienten mit einem primären kutanen Melanom besitzt. Ein therapeutischer Wert der SLN-Biopsie konnte bisher nicht bestätigt werden, sodass bei Positivität des SLN weiterhin die elektive Lymphknotendissektion zu empfehlen ist.

\section{Prä- und intraoperative Identifikation des SLN}

Präoperativ erfolgt die Identifikation des SLN durch eine Lymphabflussszintigrafie. Im Abstand von $5 \mathrm{~mm}$ wird in die Tumorumgebung bzw. Exzisionsnarbe kolloidales Technetium $99 \mathrm{~m}$-markiertes Humanalbumin (Nanokoll) intradermal injiziert. 1 bis 6 Stunden nach Injektion werden planare Szintigramme in mindestens 2 Ebenen unter Abdeckung der Injektionsstelle angefertigt. In den Frühaufnahmen kommt es zur Darstellung einer Lymphbahn, die in einen Lymphknoten (LK) mündet. Der erste speichernde LK, in dem eine Abstrombahn mündet, entspricht dem SLN (0 Abb. 1).

In der Spätaufnahme werden nur noch einzelne LK und keine Abstrombahn sichtbar.

Mithilfe der Szintillationsmesssonde markiert der Nuklearmediziner den LK auf der Haut. Der Operateur misst in dem markierten Hautareal mit einer Gamma-Messsonde nach ( $\bullet$ Abb. 2). Das Punctum maximum der Speicherung wird mit dem Zählwerk festgehalten [5-9]. An der markierten Stelle erfolgt ein kleiner Hautschnitt und mit der Gamma-Sonde wird der signalgebende LK aufgesucht und exstirpiert. Dieser LK wird mit der Sonde nachgemessen und die radioaktiven Zerfallsimpulse mit den präoperativen Werten verglichen. Sind die Werte identisch, handelt es sich bei dem entnommenen LK um den SLN, der sofort in eine Formalinlösung eingelegt wird $[10,11]$. 

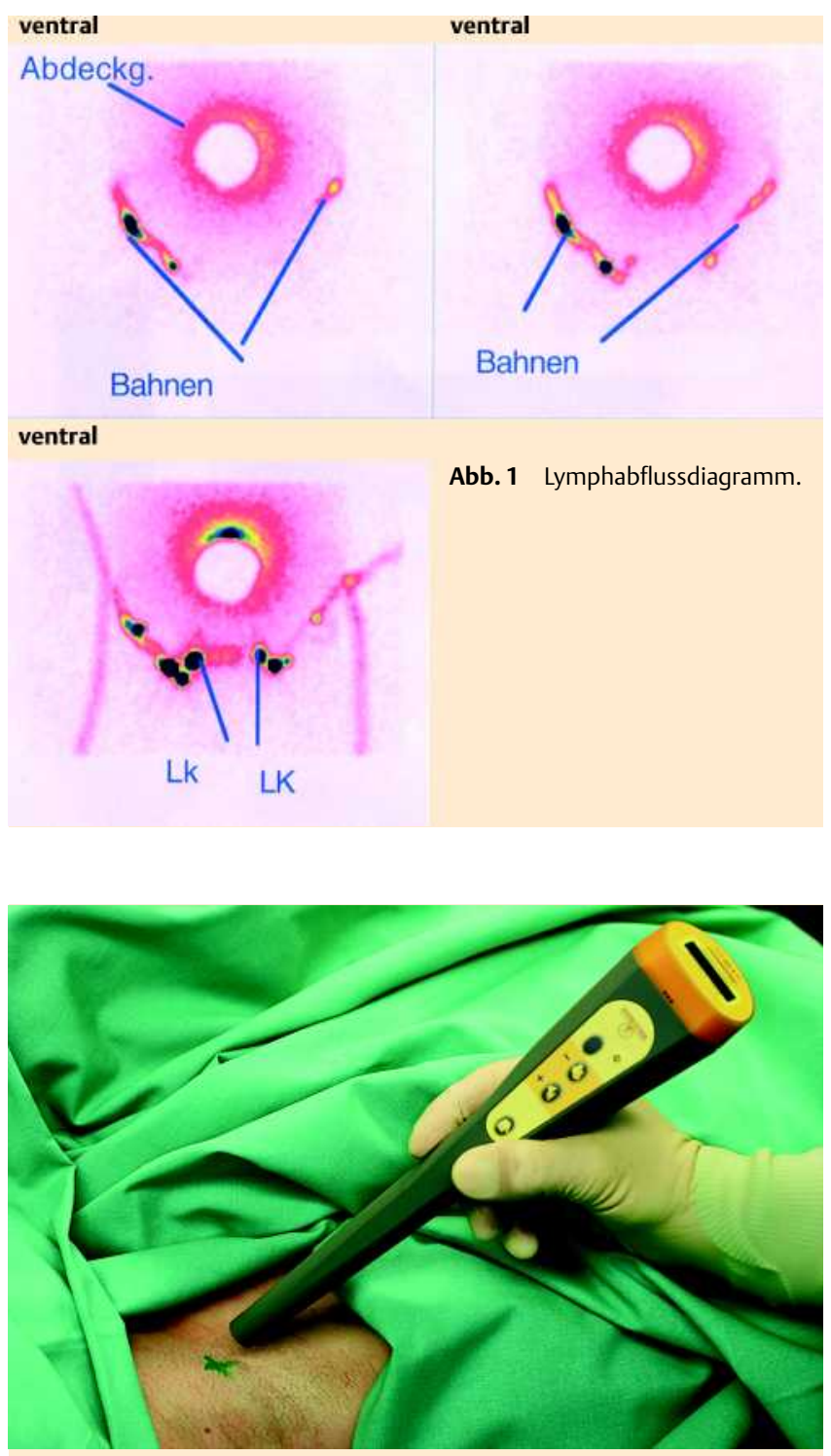

Abb. 2 Präoperative Ortung des SLN mit Gammamesssonde.

\section{Histologische Untersuchung des SLN}

Für den Erfolg der SLN-Methode wurden im „Augsburger Consensus" international und auch interdisziplinär Standards erarbeitet.

Unmittelbar nach Operation wird der SLN für $24 \mathrm{~h}$ in 4,5-prozentigem phosphatgepufferten Formalin (pH 7,0) fixiert [12].

Im dermatohistologischen Labor wird anschließend der SLN parallel zur Längsachse in $1 \mathrm{~mm}$ dünne Gewebsscheiben lamelliert und in Paraffin eingebettet.

Bei Nachweis von Tumorzellen wird ihre maximale Distanz zum Innenrand der LK-Kapsel und auch die Anzahl der befallenen LKScheiben dokumentiert [13-15].

Beim malignen Melanom sollten pro Lymphknotenscheibe eine HE-Färbung und mindestens zwei immunhistochemische Markierungen erfolgen. Als Screeningmarker werden in den immunhistochemischen Untersuchungen Anti-S-100 und HMB45 eingesetzt. Im Zweifel wird zusätzlich mit Melan A (MART1-AK) oder mit Tyrosinase-RT-PCR markiert [16-18].
Ergebnisse der SLN-Biopsie beim malignen Melanom Betrachtet man die Untersuchungsergebnisse der SLN-Biopsie beim malignen Melanom der Arbeitsgruppen Krag, Pijper und Bachter, so konnten bei den Patienten in 98 bis 100\% ein SLN mithilfe der Gammasonde intraoperativ identifiziert werden.

Der Anteil der Patienten mit Nachweis von Metastasen im SLN schwankt zwischen 12 bis $21 \%[6,7,9]$.

Dies bestätigt uns, dass die Entfernung des SLN eine Methode mit hoher Sensitivität darstellt. In weiteren Studien wird auch die klinische Relevanz von molekularbiologisch nachgewiesenen Tumorzellen beim Melanom deutlich.

Studien von Bostick und Blaheta konnten bei Patienten mit histologisch positiven SLN die höchsten Rezidivraten (31-67\%) nachweisen. Patienten mit histologisch negativen SLN zeigten mit 0 bis $6 \%$ eine deutlich niedrigere Rezidivrate bzw. rezidivfreies Überleben $[17,19,20]$.

Der Tumorzellnachweis im SLN besitzt somit eine klinische Relevanz hinsichtlich einer Tumorprogression. Die Patienten mit positiven Lymphknotenbefunden werden anschließend einer regionalen Lymphknotendissektion zugeführt.

Ergebnisse von Konz et al. zeigen auch, dass mit zunehmender Tumordicke beim malignen Melanom ein Anstieg von lymphogenen Metastasen in das regionale Lymphabflussgebiet besteht. Patienten mit einer Tumordicke von 1-1,5 mm wiesen einen positiven SLN in $15 \%$ und Patienten mit einer Tumordicke über $3 \mathrm{~mm}$ in $60 \%$ der Fälle auf [11].

Bei jeder Methode, so auch bei der SLN-Biopsie, gibt es Fehlerquellen. Die LK-Aufarbeitung ist noch relativ wenig standardisiert, sodass Mikrometastasen im SLN häufig nicht aufgefunden werden. Dies ist eindeutig abhängig von der Untersuchungstechnik (Anzahl der Schnitte, HE-Färbung, immunhistologische Färbung, RT-PCR).

Auch die Unerfahrenheit des Operateurs stellt eine Fehlerquelle dar. Mehrere klinische Studien zeigten, dass ein Operateur erst nach über 50 durchgeführten SLN-Biopsien eine Detektionsrate von über $90 \%$ erreicht $[3,13]$.

Entscheidend für den Erfolg der SLN-Biopsie ist auch die enge Zusammenarbeit mit dem Nuklearmediziner und dem Operateur.

Die Arbeitsgruppe Morton untersuchte in einer sehr umfangreichen klinischen Studie das krankheitsfreie Überleben sowie Gesamtüberleben bei Patienten mit malignem Melanom. Eindeutig konnte bei Patienten, die einen negativen SLN aufwiesen (90,2\%) eine höhere Erkrankungsfreiheit gegenüber Patienten mit positiven SLN $(72,2 \%)$ bestätigt werden. Somit stellt die SLN-Biopsie einen wichtigen diagnostischen Marker dar [21,22].

Im Vergleich Patientengruppe mit SLN-Biopsie versus Patientengruppe Beobachtung (ohne SLN-Biopsie) bestand ein signifikanter Unterschied. In der SLN-Biopsiegruppe waren $5 \%$ weniger Metastasen nachweisbar als in der Beobachtungsgruppe. Daraus könnte man schlussfolgern, dass eine SLN-Biopsie das Gesamtüberleben verlängert und somit auch einen therapeutischen Wert besitzt. Sieht man sich aber die Untersuchungsergebnisse des Gesamtüberlebens SLN-Biopsiegruppe versus Beobachtungsgruppe an, so wird dies nicht bestätigt. Ein signifikanter Unterschied besteht nicht.

Ein therapeutischer Wert der SLN-Biopsie konnte nicht bewiesen werden, jedoch der eindeutige prognostische Faktor dieser Methode. 


\section{Schlussfolgerungen}

Die SLN-Biopsie ist eine minimalinvasive diagnostische Methode, die ein exaktes Staging der dem Primärtumor zugehörigen Lymphknotenregion ermöglicht. Eine genaue Lokalisation des SLN ist durch die präoperative Lymphabflussszintigrafie und durch die intraoperative Gammasondenmessung möglich.

Diese Methode weist bei fachgerechter Anwendung eine Sensitivität von nahezu $100 \%$ auf.

Die SLN-Biopsie hat einen potenziellen diagnostischen Wert und ist bereits eine Routinemaßnahme in der Ausbreitungsdiagnostik.

Wird ein positiver SLN nachgewiesen, so handelt es sich um einen Hochrisikopatienten mit massiver Abnahme der 5-JahresÜberlebensrate. Diese Patienten werden anschließend einer therapeutischen regionalen LK-Dissektion zugeführt.

Patienten mit negativen SLN bleibt eine radikale LK-Dissektion, die relativ hohe Komplikationsraten aufweist, erspart.

Durch die SLN-Biopsie ist es nun möglich, auf die ungezielte prophylaktische LK-Dissektion zu verzichten.

\section{Abstract}

\section{The Role of Sentinel Lymph Node Biopsy of Malignant Melanoma \\ $\nabla$}

The sentinel lymph node biopsy is regarded as a reliable staging method and should be performed with patients with a malignant melanoma starting from a tumour thickness of $1 \mathrm{~mm}$ onwards.

The relapse-free survival time of the patients with a melanoma clearly correlates with the status of the sentinel lymph node (SLN).

It has been shown in numerous clinical studies that the SLN holds an important diagnostic and prognostic significance for patients with primary cutaneous melanoma. A therapeutical value of the SLN biopsy could not be confirmed so far, so that in case of the positiveness of the SLN the elective dissection of a lymph node still has to be recommended.

\section{Literatur}

1 Morton DL, Wen DR, Wong JH et al. Technical details of intraoperative lymphatic mapping for early melanoma. Arch Surg 1992; 127: 392 399

2 Bachter D. Ist die Entfernung des Sentinellymphknotens bei nicht melanozytären Hautmalignomen sinnvoll? In: Rompel R, Petres J (Hrsg). Fortschritte der Operation und onkologischen Dermatologie. Stuttgart: Thieme, 2002: 18

3 Cochrane AJ, Balda B-R, Starz H et al. The Augsburg Consensus. Techniques of lymphatic mapping, sentinel lymphadenectomy and com- pletion lymphadenectomy in cutaneous malignancies. Cancer 2000; 80: $236-241$

4 Düker J, Starz H, Bachter D, Balda B-R. Prognostic and therapeutic implications of sentinel lymphonodectomy and S-staging in Merkel cell carcinoma. Dermatology 2001; 202: 225 - 229

5 Alex JC, Krag DN. Gamma-probe-guided localization of lymph nodes. Surg Oncol 1993; 2: 137-144

6 Bachter D, Balda BR, Vogt H, Buchels H. Die „Sentinel“ Lymphonodektomie mittels Szintillationsdetektor. Eine neue Strategie in der Behandlung maligner Melanome. Hautarzt 1996; 47: 754-758

7 Bachter D, Goerdt S. Präoperative Identifikation und chirurgische Entfernung des Sentinel-Lymphknotens bei malignen Hauttumoren. Akt Dermatol 2002; 28: 265-272

8 Morton DL, Wen DR, Cochran AJ. Management of early-stage melanoma by intraoperative lymphatic mapping and selective lymphadenectomy. Surg Oncol Clin North Am 1992; 1: 247 - 259

9 Pijpers R, Collet GJ, Meijer S, Hoekstra OS. The impact of dynamic lymphoszintigraphy and gamma probe guidance on sentinel node biopsy in melanoma. Eur J Nucl Med 1995; 22: 1238-1241

10 Konz B, Weiss M, Sander C. Die Sentinel-Lymph-Node-Exstirpation: Ein Fortschritt in der Melanomtherapie? Beilage im Dt Derm 1998; 46: 4 10

11 Konz B, Weiss M, Sander CA, Volkenandt M, Hahn K, Plewig G. SentinelLymph-Node-Exstirpation. Münch Med Wschr 1998; 22: 320-325

12 Starz H, Cochran AJ, Balda B-R. Die Sentinel-Lymphonodektomie aus histologischer Sicht. Akt Dermatol 2002; 28: 273-278

13 Cochrane AJ, Starz H. Pathologic aspects. In: Cody HS III (ed). Sentinel Lymph Node Biopsy. London: Martin Dunitz Ltd, 2002: 79-90

14 Starz H. Suspected diagnosis: malignant melanoma. In: Starz H. Immunohistochemistry on Paraffin sections: Technical Principles and Diagnostic Applications in Routine Pathology. Darmstadt: GJT, 1991: $42-43$

15 Starz H, Flaig MJ, Sander CA. Histologie des Sentinel-Lymphknotens. In: Volkenandt M, Plewig G (Hrsg). Maligne Melanome, Empfehlungen zur Diagnostik, Therapie und Nachsorge. München: Zuckschwerdt, 2000: 32 - 35

16 Cochrane AJ, Wen D-R, Herschman HR. Occult melanoma in lymph nodes detected by antiserum to S-100 protein. Int J Cancer 1984; 34 : $159-163$

17 Bostick PJ, Morton DL, Turner RR et al. Prognostic significance of occult metastases detected by sentinel lymphadenectomy and reverse transcriptasepolymerase chain reaction in early stage melanoma patients. J Clin Oncol 1999; 17: 3238 - 3244

$18 \mathrm{Li}$ W, Stall A, Shivers SC et al. Clinical relevance of molecular staging for melanoma: comparison of RT-PCR and immunhistochemistry staging in sentinel lymph nodes of patients with melanoma. Ann Surg 2000; 231: $795-803$

19 Blaheta HJ. Sentinel Node Biopsy: Impact on Prognosis and Treatment. Akt Dermatol 2002; 28: 279-284

20 Blaheta HJ, Ellwanger U, Schittek B et al. Examination of regional lymph nodes by sentinel node biopsy and molecular analysis provides new staging facilities in primary cutaneous melanoma. J Invest Dermatol 2000; 114: 637-642

21 Morton DL, Cochrane AJ, Thompson JF et al. Sentinel Node Biopsy for Early-Stage Melanoma. Ann Surg 2005; 242: 302 - 313

22 Morton DL, Thompson JF, Cochran AJ et al. Sentinel-Node Biopsy or Nodal observation in Melanoma. N Engl Med 2006; 13: 1307-1315 